Full length article

\title{
Tissue response to collagen containing polypropylene meshes in an ovine vaginal repair model
}

\author{
Saeedeh Darzi ${ }^{\mathrm{a}, \mathrm{b}}$, Iva Urbankova ${ }^{\mathrm{c}}$, Kai Su ${ }^{\mathrm{d}}$, Jacinta White ${ }^{\mathrm{d}}$, Camden Lo ${ }^{\mathrm{a}}$, David Alexander ${ }^{\mathrm{d}}$, \\ Jerome A. Werkmeister ${ }^{\mathrm{b}, \mathrm{d}, 1}$, Caroline E. Gargett ${ }^{\mathrm{a}, \mathrm{b}, *, 1}$, Jan Deprest ${ }^{\mathrm{c}, 1}$ \\ ${ }^{a}$ Hudson Institute of Medical Research, 27-31 Wright Street, Clayton, Victoria 3168, Australia \\ ${ }^{\mathrm{b}}$ Department of Obstetrics and Gynaecology, Monash University, Clayton, Victoria 3168, Australia \\ ${ }^{\mathrm{C}}$ Centre for Surgical Technologies and Department of Development and Regeneration, KU Leuven, Leuven, Belgium \\ d CSIRO Manufacturing, Bayview Avenue, Clayton, Victoria 3169, Australia
}

\section{A R T I C L E I N F O}

\section{Article history:}

Received 24 November 2015

Received in revised form 27 April 2016

Accepted 3 May 2016

Available online $\mathrm{xxxx}$

\section{Keywords:}

Pelvic Organ prolapse

Ovine model

Macrophage response

Collagen tissue regeneration

Birefringence

\begin{abstract}
A B S T R A C T
Pelvic Organ Prolapse (POP) is the herniation of pelvic organs into the vagina. Despite broad acceptance of mesh use in POP surgical repair, the complication rate is unacceptable. We hypothesized that collagencontaining polypropylene (PP) mesh types could modulate mesh-tissue integration and reduce long-term inflammation, thereby reducing mesh-associated complications. This study compared the long-term tissue response to an unmodified PP mesh and two collagen containing meshes in an ovine model which has similar pelvic anatomy and vaginal size to human. Three commercially available macroporous PP meshes, uncoated PP mesh (Avaulta Solo)(PP), the same textile PP mesh layered with a sheet of cross-linked porcine acellular matrix (Avaulta Plus) (PP-ACM) and a different yet also macroporous PP (Sofradim) mesh coated with solubilized atelocollagen (Ugytex) (PP-sCOL) were implanted in the ovine vagina and tissue explanted after 60 and 180 days. The macrophage phenotype and response to implanted meshes, and vascularity were quantified by immunostaining and morphometry. We quantified changes in extracellular matrix composition biochemically and collagen organisation and percentage area around the interface of the mesh implants by Sirius Red birefringence and morphometry. PP-ACM induced a more sustained inflammatory response, indicated by similar $\mathrm{CD} 45^{+}$leukocytes but reduced $\mathrm{CD} 163^{+} \mathrm{M} 2$ macrophages at 60 days $(\mathrm{P}<0.05)$. PPsCOL increased Von Willebrand Factor (vWF)-immunoreactive vessel profiles after 60 days. At the micromolecular level, collagen birefringence quantification revealed significantly fewer mature collagen fibrils (red, thick fibrils) at the mesh-tissue interface than control tissue for all mesh types $(\mathrm{P}<0.001)$ but still significantly greater than the proportion of immature (green thin fibrils) at 60 days $(\mathrm{P}<0.05)$. The proportion of mature collagen fibrils increased with time around the mesh filaments, particularly those containing collagen. The total collagen percent area at the mesh interface was greatest around the PP-ACM mesh at 60 days $(P<0.05)$. By 180 days the total mature and immature collagen fibres at the interface of the mesh filaments resembled that of native tissue. In particular, these results suggest that both meshes containing collagen evoke different types of tissue responses at different times during the healing response yet both ultimately lead to physiological tissue formation approaching that of normal tissue.
\end{abstract}

\section{Statement of Significance}

Pelvic organ prolapse (POP) is the descent of the pelvic organs to the vagina. POP affects more than $25 \%$ of all women and the lifetime risk of undergoing POP surgery is $19 \%$. Although synthetic polypropylene (PP) meshes have improved the outcome of the surgical treatment for POP, there was an unacceptable rate of adverse events including mesh exposure and contracture. It is hypothesized that coating the PP meshes with collagen would provide a protective effect by preventing severe mesh adhesions to the wound, resulting in a better controlled initial inflammatory response, and diminished risk of exposure. In this study we assessed the effect of two collagen-containing PP meshes on the long-term vaginal tissue response using new techniques to quantify these tissue responses.

(c) 2016 Acta Materialia Inc. Published by Elsevier Ltd. All rights reserved.

\footnotetext{
* Corresponding author at: The Ritchie Centre, Hudson Institute of Medical Research, 27-31 Wright Street, Clayton, Victoria 3168, Australia.

E-mail address: caroline.gargett@hudson.org.au (C.E. Gargett).

1 Equal senior authors.
} 


\section{Introduction}

Pelvic organ prolapse (POP) is defined as the downward descent or herniation of pelvic organs into the vagina [1]. Common symptoms of POP are pressure or bulge in the pelvic area, urinary incontinence and sexual dysfunction [2]. POP affects more than $25 \%$ of all women and it is estimated that the lifetime risk of undergoing surgery for POP is $19 \%[3,4]$. Reconstructive surgery is the main treatment for POP and augmentation using synthetic polypropylene (PP) biomaterial meshes has improved the outcome of the surgical treatment for POP [5], although this is considered controversial. Lightweight, macro porous monofilament PP meshes were the most common non-degradable synthetic material used in transvaginal POP surgery as these showed lower foreign body tissue responses and reduced material stiffness [6]. While these mesh implants resulted in greater cure rates than native tissue surgery, there was an unacceptable rate of adverse events [7-9] including mesh exposure and contracture. The Food and Drug Administration (FDA), and later in Europe the Scientific Committee on Emerging and Newly Identified Health Risks (SCENIHR) issued warnings on the transvaginal placement of synthetic implants [10], resulting in companies withdrawing some mesh products from the market. New guidelines from the FDA and the International Urogynecological Association (IUGA) recommend testing new meshes in preclinical models to improve outcomes for any new mesh products being developed before translation to the clinic [11].

In addition, although mesh implantation induces a proinflammatory response, which is followed by fibrosis with a concomitant increase in the strength of surgical repair, this fibrotic response can cause pain and discomfort. In order to minimize fibrosis and pain, many investigations have examined the modification of mesh designs including, pore size (large versus small pore size), weight (lightweight or heavyweight) and collagen coating [12]. One approach to improve the biocompatibility of mesh was to coat the mesh with extracellular matrix (ECM) proteins. ECM coated meshes are degradable and can allow tissue remodelling and formation of appropriate soft tissue rather than fibrosis by inducing angiogenesis and promoting the accumulation of progenitor cells at the site of implantation [13]. It has been reported that coating the PP mesh with acellular porcine collagen resulted in less erosion and inflammation. It was hypothesized that the collagen would provide a protective effect by preventing severe mesh adhesions to the wound thereby decreasing direct mesh-tissue contact with the polymer, resulting in a better controlled initial inflammatory response, and diminished risk of exposure [14,15].

The host response to surgically implanted biomaterials is a critical determinant of its success or failure [16]. The early tissue response to a synthetic mesh is acute inflammation, characterised by an influx of neutrophils followed by pro-inflammatory (referred to as M1) macrophages. After the acute response following departure of the neutrophils, chronic inflammation develops that can vary in time and extent, particularly the rate at which M1 macrophages differentiate into an anti-inflammatory M2 macrophage phenotype, which will ultimately influence the wound healing response and the quality of new tissue formation [16].

An appropriate animal model is necessary to test new meshes to fulfil the IUGA requirement for preclinical studies. Although rodents are widely used because of their low cost and ease to work with, the small size of their vagina has prompted the recent shift to larger animal models [14,17]. The ovine model is attractive because the pelvis has similar anatomy and size as the human pelvis and has similar pelvic support structures as in women $[18,19]$. Ewes also have prolonged labours with relatively large foetuses and may also spontaneously develop postpartum POP [20,21]. The limitation of the ovine model is their quadrupedal posture, which reduces intra-abdominal pressure experienced by the pelvis. However, it has been suggested that their ruminant physiology and tendency to ruminate facing uphill increases the pressure on the pelvic structures including the vaginal wall [19]. Moreover, their large size and hence vaginal capacity allows the examination of mesh with dimensions more representative of those implanted into women. In addition, several meshes can be implanted into the same animal [14].

The objective of this study was to evaluate whether the format of collagen coating on the PP mesh affects the chronic inflammatory host response and healing process in the long term to the implanted mesh. Sixty days was chosen to examine the tissue response to the effect of differential degradation of the collagen coating between the two modified PP meshes that used two different collagen formats and 180 days was chosen as a longer term steady state end point to examine if any differences were still apparent. We assessed the effect of two collagen-containing PP meshes on the long-term vaginal tissue response, evaluating the chronic inflammatory macrophage response, vascular response, the total collagen and glycosaminoglycans (GAG) content, and the organisation and density of collagen at the mesh-tissue interface in explanted vaginal tissues. We used standard techniques to measure these parameters and a new technique to quantify the collagen response at the mesh tissue interface by quantifying red (mature) and green (immature) collagen fibril deposition by assessing birefringence in 100 and $200 \mu \mathrm{m}$ increments in an ovine model.

\section{Methods}

\subsection{Mesh}

Three commercially available so called Amid-I macroporous PP meshes were used where the PP component was the same in all; 1. Avaulta Solo (PP) (Bard Medical, Convington, GA, USA, $58 \mathrm{~g} / \mathrm{m}^{2}$ ), 2. Avaulta Plus (PP-ACM) (composed of the same fabric as PP with a hydrophilic slowly degrading cross-linked porcine acellular matrix (ACM) sheet comprising collagen, $0.5 \mathrm{~mm}$ thick and $1.8 \mathrm{~mm}$ pores, $100 \mathrm{~g} / \mathrm{m}^{2}$ ) and 3. PP-sCOL (PP-sCOL) (Sofradim International, Trevoux, France, $38 \mathrm{~g} / \mathrm{m}^{2}$ ), comprising PP monofilaments coated with reconstituted enzyme-purified solubilized atelocollagen, polyethylene glycol and glycerol (Supplementary Fig. 1)

\subsection{Animal implantation}

Animal surgery and mesh implantation was undertaken at the KU Leuven in accordance with protocols approved by the local Animal Ethics Committee as previously reported [17]. Thirty-six multiparous Texel ewes underwent surgical implantation of mesh ( $n=12 /$ mesh type). Briefly, following general anaesthesia, antibiotic prophylaxis, and hydro-dissection of the rectovaginal space, a single vaginal incision was made and a $35 \times 35 \mathrm{~mm}$ flat mesh was inserted and fixed to underlying tissue of the posterior wall with multiple interrupted 4/0 polypropylene sutures (Prolene; Ethicon, Zaventem, Belgium). We chose $35 \times 35 \mathrm{~mm}$ mesh size to avoid graft related complications associated with implanting larger mesh sizes, including exposure and contraction [9]. The vaginal incision was closed with continuous 2/0 polyglactin 910 (Vicryl; Ethicon). Tissues were explanted after 60 and 180 days and processed as previously described to assess the effect of collagen after partial/complete resorption of collagen on the collagen-containing meshes and the chronic rather than acute inflammatory response [17]. Control tissues were collected from non-operated regions adjacent to the implants and at the same distance from the introitus as there are regional differences cranio-caudally and between 
the anterior and posterior vaginal wall in sheep [22,23]. The $1.0 \times 0.5 \mathrm{~cm}$ explants were fixed in formalin and embedded in paraffin or embedded in OCT and then frozen. Frozen and paraffin blocs were cut into $5 \mu \mathrm{m}$ sections at the Hudson Institute. The gross anatomical outcomes and biomechanical evaluation of explants of these animals was already reported on before [24]. In cases of gross anatomic graft related complications (GRC) which was low (areas with exposure and local inflammation), outcome measurements described below were scored in areas away from GRC to ensure focus on the effects of collagen-coating were not complicated by a different type of inflammatory reaction to exposed mesh.

\subsection{Immunohistochemical and histomorphometric analysis of explanted tissues}

Frozen $5 \mu \mathrm{m}$ sections were thawed and fixed with pre cooled acetone and then washed with PBS. Endogenous peroxidase was quenched with $0.3 \% \mathrm{v} / \mathrm{v} \mathrm{H}_{2} \mathrm{O}_{2}$ followed by protein blocking (Protein Block serum free, Dako, USA) for $30 \mathrm{~min}$ at RT. After three washes in PBS, primary antibodies (CD45, CD163, CD206, vWF, aSMA) were incubated for $1 \mathrm{~h}$ at 37C; isotype matched IgG antibodies were used as negative controls and applied at the same concentration (Table 1). HRP-labelled polymer (Dako, USA) conjugated anti-mouse secondary antibody was incubated for $30 \mathrm{~min}$ at RT. Haematoxylin was used for nuclear staining and the slides were coversliped with mounting medium (Dako, USA). Images (2-6/ section) were taken of immunostained sections for CD45, CD163, CD206, aSMA and vWF for each explant at both time-points using a Leica-DMR Microscope at 10x magnification and the images were analysed using Metamorph software (Molecular Devices, LLC) to measure positive staining around mesh filaments in $100 \mu \mathrm{m}$ increments as described previously [25]. Briefly, individual filaments were outlined manually and the software counted the signal area around mesh filaments. The positive signal area (pixels) for every image in first $100 \mu \mathrm{m}$ increment for CD45, CD163, and CD206, and the first $250 \mu \mathrm{m}$ for $\alpha \mathrm{SMA}$ and $\mathrm{vWF}$ was recorded and divided by the total tissue area examined and the average calculated per ovine sample and this was used for statistical analysis.

\subsection{Biochemical analysis of total collagen and GAG content}

Total collagen was determined from the supernatant of papaindigested tissue explants from implanted areas and control tissue areas, as previously described [26]. Briefly, frozen tissue explants were freeze dried for $6 \mathrm{~h}$, weighed and digested in $1 \mathrm{~mL}$ papain digestion buffer $\left(0.1 \mathrm{M} \mathrm{Na}_{2} \mathrm{HPO}_{4}, 5 \mathrm{mM}\right.$ EDTA, $5 \mathrm{mM}$ Cysteine $\cdot \mathrm{HCl}$, $\mathrm{pH} 7.4$ ) containing $0.5 \mathrm{mg} / \mathrm{mL}$ papain for $12-16 \mathrm{~h}$ at $60 \mathrm{C}$. Following digestion, samples were centrifuged at $13,200 \mathrm{rpm}$ for $10 \mathrm{~min}$ and $0.5 \mathrm{~mL}$ of supernatant hydrolysed in $6 \mathrm{~N} \mathrm{HCl}$ at $115 \mathrm{C}$ for $4 \mathrm{~h}$. Hydroxyproline (Hyp) was measured spectrophotometrically at $560 \mathrm{~nm}$ after reaction with $0.05 \mathrm{~mol} / \mathrm{L}$ chloramine-T (Sigma) and $10 \%(\mathrm{w} / \mathrm{v}$ in 2-methoxyethanol) P-dimethylaminobenzaldehyde (Sigma). A standard curve using L-hydroxyproline $(0-10 \mathrm{mg} / \mathrm{mL})$ (Sigma) was used to calculate the Hyp concentration. Total collagen was calculated using a hydroxyproline to collagen ratio of 0.143:1 [27].

Sulphated GAG was measured by reacting $40 \mu \mathrm{L}$ of the papaindigest supernatant with $1 \mathrm{~mL}$ dimethylmethylene blue (DMMB) reagent solution ( $40 \mathrm{mM} \mathrm{NaCl}, 40 \mathrm{mM}$ glycine, $0.1 \mathrm{M} \mathrm{HCl}, 46 \mathrm{mM}$ DMMB (Sigma), pH 3.0) [28]. GAG content was measured by absorbance at $525 \mathrm{~nm}$ using a UV-vis spectrophotometer. A standard curve was created using 0.001 and $0.02 \mathrm{mg}$ of whale cartilage chondroitin sulphate A (Sigma) dissolved in PBS and used to determine sulphated GAG concentration.
Table 1

Antibodies used for Immunostaining.

\begin{tabular}{|c|c|c|c|}
\hline Primary antibodies & $\begin{array}{l}\text { Concentration } \\
(\mu \mathrm{g} / \mathrm{mL})\end{array}$ & Isotype & Supplier \\
\hline CD45 & $(0.5 \mu \mathrm{g} / \mathrm{mL}) 1 / 200$ & $\begin{array}{l}\text { Mouse } \\
\text { IgG1 }\end{array}$ & $\begin{array}{l}\text { AbD } \\
\text { Serotec }\end{array}$ \\
\hline $\begin{array}{l}\text { CD163 (M2 macrophage } \\
\text { marker) }\end{array}$ & $(0.5 \mu \mathrm{g} / \mathrm{mL}) 1 / 200$ & $\begin{array}{l}\text { Mouse } \\
\text { IgG1 }\end{array}$ & $\begin{array}{l}\text { AbD } \\
\text { Serotec }\end{array}$ \\
\hline $\begin{array}{l}\text { CD206 (M2 macrophage } \\
\text { marker) }\end{array}$ & $(0.5 \mu \mathrm{g} / \mathrm{mL}) 1 / 200$ & $\begin{array}{l}\text { Mouse } \\
\text { IgG1 }\end{array}$ & Dendritics \\
\hline $\begin{array}{l}\text { A-Smooth Muscle Actin } \\
\text { ( } \alpha \text { SMA) }\end{array}$ & $1 / 400$ & $\begin{array}{l}\text { Mouse } \\
\lg 2 a\end{array}$ & Dako \\
\hline Von Willebrand Factor (vWF) & $1 / 50$ & $\begin{array}{l}\text { Mouse } \\
\text { IgG1 }\end{array}$ & Dako \\
\hline
\end{tabular}

\subsection{Collagen alignment by Sirius red birefringence image analysis}

Paraffin sections of control tissue (distal tissue away from the mesh) and implant (mesh and tissue complex) were dewaxed and rehydrated in graded alcohols and stained with Sirius Red F3B $(0.1 \mathrm{~g} / 100 \mathrm{~mL}$ saturated picric acid solution) (Sigma-Aldrich, USA) for $1 \mathrm{~h}$ at room temperature (RT) to determine collagen alignment. For morphometric analysis, the stained slides were washed in running water (non-acidified) to remove the yellow picric acid counterstain. Sirius red stained sections were observed under a light microscope (Olympus BX61) (Fig. 1A). Using polarizing filters, the stained collagen fibres showed a mixed proportion of birefringent staining patterns ranging from green to yellow to orange/red, depicting immature to mature collagen fibril organisation, respectively (Fig. 1B). Threshold gating was set to segregate the polarized images into two distinct colours, red to capture the thicker mature fibrils and green to capture the thinner immature less organised fibril. The staining patterns were captured digitally and image segmentation of pixel area and intensity converted into areas of green and red as described below (Fig. 1C, E) and their relative proportion was measured by Metamorph software in 100 (Fig. 1D) and 200 (101-200) $\mu \mathrm{m}$ (Fig. 1F) increments around the mesh fibres.

\subsection{Metamorph software}

Image analysis was undertaken with MetaMorph v7.7.0.0. A user-defined region-of-interest (ROI) corresponding to the perimeter boundary of the mesh is used as the basis of the analysis. The perimeter of this ROI was processed using the Euclidean distance routine to establish a radial distance map of the tissue relative to the mesh. From this radial distance map, two band regions were established corresponding to a radial distance of $1-100 \mu \mathrm{m}$ and 101-200 $\mu \mathrm{m}$ away from the perimeter of the ROI. Each band region was converted to binary masks and applied back to the original image to the corresponding isolate tissue areas, which was then thresholded for both green and red channels separately to measure both area and optical density. Thresholding criteria were selected as the upper $80 \%$ of the 8 -bit linear signal range for each channel.

\subsection{Statistical analysis}

GraphPad Prism 6 was used for statistical analysis. Results are reported as mean \pm SEM for each experimental group $(n=6$ meshes/group/time point).

For birefringence analysis ANOVA was performed using R software. $T$ tests for identifying differences between collagen levels in specific groups were calculated using estimates for each variance component derived from the ANOVA. The Pearson Chi-Square Normality Test and The D'Agostino Omnibus Test was used to assess the normal distribution of data and found not significant. We therefore used parametric analyses. 

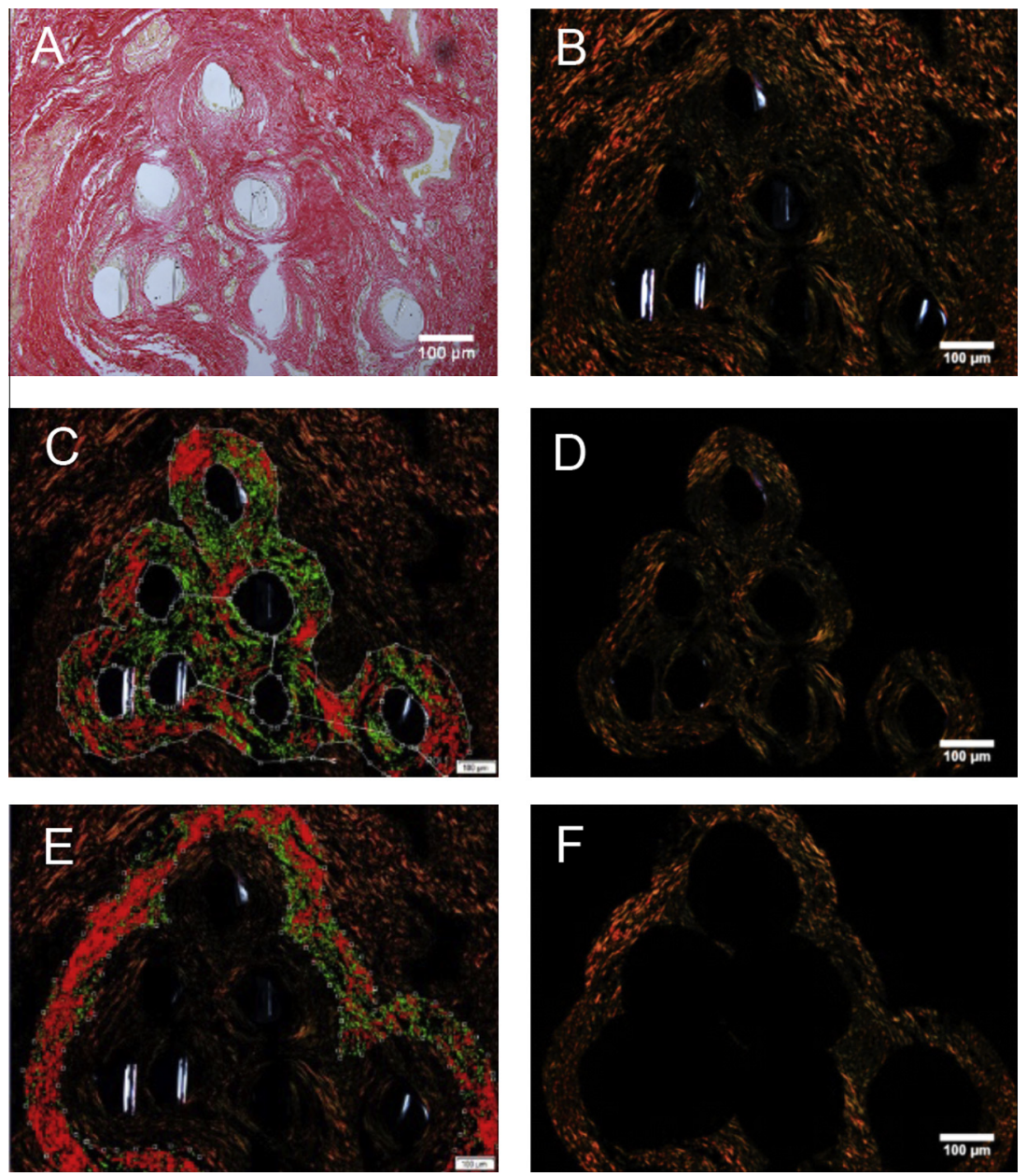

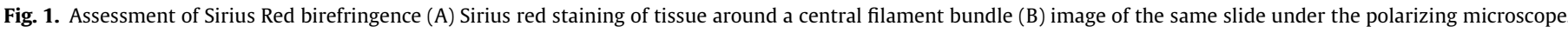

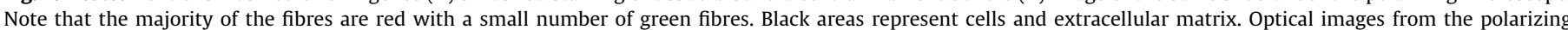

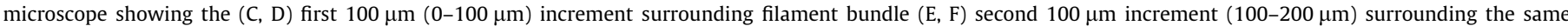
filament bundle. (For interpretation of the references to colour in this figure legend, the reader is referred to the web version of this article.)

\section{Results}

\subsection{Inflammatory response to implanted collagen containing $P P$ meshes}

Immunohistochemistry was used to examine the chronic cellular response to the two implanted collagen containing polypropylene meshes and the unmodified PP mesh in the ovine vagina. The pan CD45 leukocyte marker showed that these inflammatory cells were concentrated around the individual PP mesh filaments and filament clusters in a similar pattern for all 3 meshes at both 60 and 180 days implantation (Fig. 2.1A-F). Image analysis quantification of the first $100 \mu \mathrm{m}$ increments around individual mesh filaments showed no difference in $\mathrm{CD} 45^{+}$leukocyte density between mesh types after 60 days or 180 days (Fig. 2.1G). Since CD45 encompasses all inflammatory cell types associated with foreign mesh implants, we next investigated the proportion of tissue regenerative $\mathrm{M} 2$ macrophages that play a key role in the late inflammatory response to synthetic meshes [26]. Immunostaining was performed with two M2 phenotypic markers, CD163 and CD206 (Fig. 2.2A-F and 2.3 A-F, respectively). Again, the level of CD163+ and CD206+ cells was quantitated in the first $100 \mu \mathrm{m}$ around the individual filaments. CD163 immunostained macrophages around PP-ACM filaments was significantly lower than around PP-sCOL at 60 days $(\mathrm{P}<0.05)$ (Fig. $2.2 \mathrm{G}$ ). No significant differences were observed between meshes at 180 days. Neither was there a difference between 60 and 180 days for any of the three meshes (Fig. 2.2G). Unlike CD163, there were no differences in the percentage CD206+ cells between the three mesh types at 60 or 180 days (Fig. $2.3 \mathrm{~A}-\mathrm{G}$ ), nor for individual meshes between 60 and 180 days.

\subsection{Vascular response to collagen-containing PP meshes}

The extent of vascularization into the mesh implanted tissues was assessed with endothelial (vWF) and vascular smooth muscle ( $\alpha$ SMA) immunostaining in the first $250 \mu \mathrm{m}$ around the PP filaments. A larger increment diameter around the filaments was examined because the vessels are more widely distributed around the mesh compared to macrophages. vWF (Fig. 3.1A-F) and $\alpha$ SMA (Fig. 3.2A-F) immunostaining showed that vessels are both between and around filament knots, and not as closely associated with filaments as the leukocytes. Monofilaments coated with collagen (PP-sCOL) showed greater numbers of small vWF+ vessel profiles within this $250 \mu \mathrm{m}$ region around the filaments compared to non-collagen coated (PP) and the collagen layer coated PP mesh (PP-ACM) after 60 days (both $\mathrm{P}<0.05$ ) (Fig. 3.1G). By 180 days the level of vascularization had diminished around PP-sCOL filaments (Fig. 3.1D-F), and there were no significant differences between any of the 3 meshes (Fig. 3.1G). aSMA immunostained 

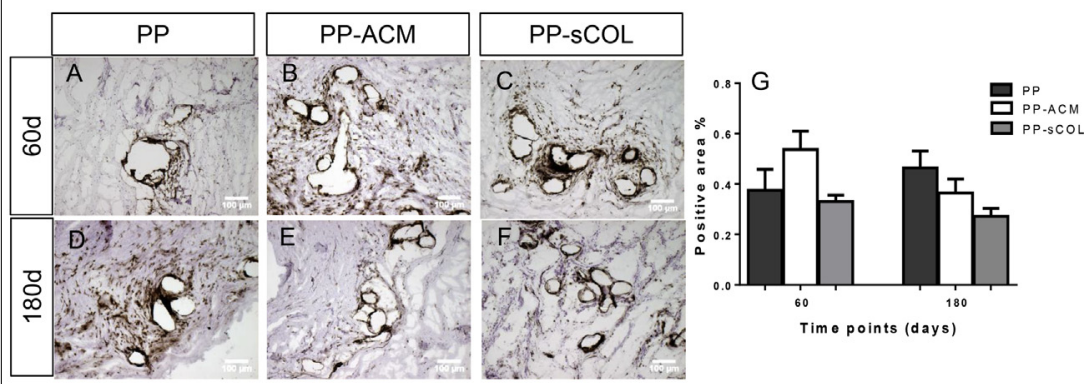

2
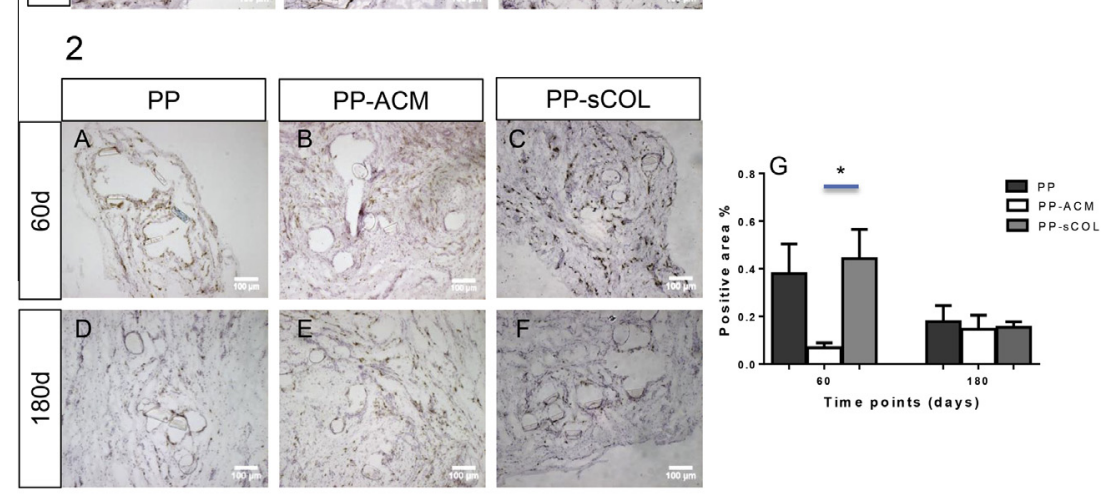

\section{3}
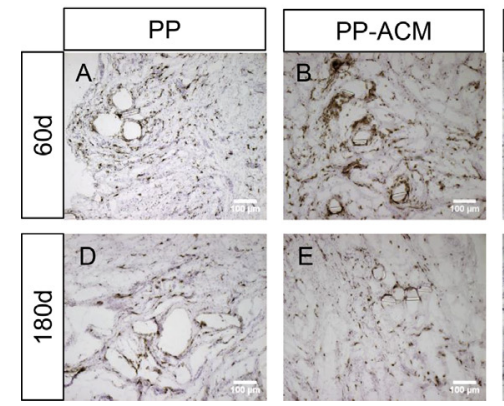
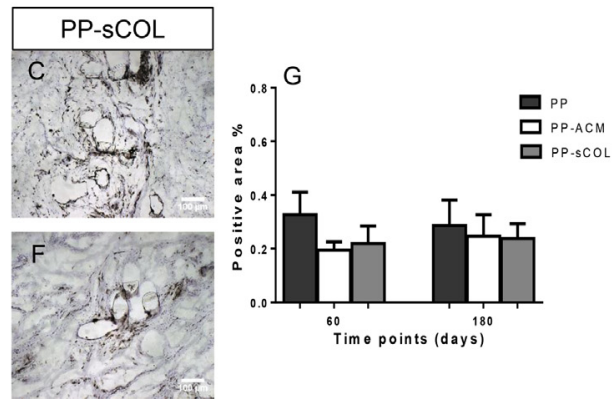

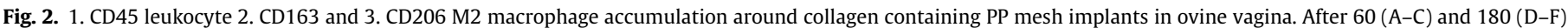

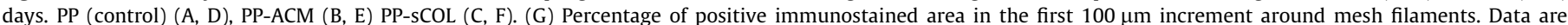
Mean \pm SEM of $n=6$ animals/group. ${ }^{*} P<0.05$.

vessel profiles appeared stronger than vWF staining and there were no significant difference between the mesh types at both time points nor for mesh types between the two time-points (Fig. 3.2A-F and G).

\subsection{Total extracellular matrix synthesis in and round implanted collagen-coated PP meshes}

Collagen and GAG are two major structural proteins and macromolecules in extracellular matrix. There was little difference in the total collagen deposition (Fig. 4A) and GAG content (Fig. 4B) between the mesh types, the control and explanted tissues or between 60 and 180 days implantation.

\subsection{Collagen composition and organisation at the interface of implanted collagen-containing meshes}

While the total ECM content, predominantly collagen, was substantially comparable between tissue from mesh implants and normal tissue, further analysis of the extent and organisation of new collagen deposition close to the mesh interface was undertaken to assess the quality of the collagen fibrils deposited as the tissue heals around the implanted mesh. Polarized light microscopy on
Picrosirius Red stained tissue (Fig. 1C) was used to quantify the level of mature (thicker fibrils with defined organised collagen packing) and immature (thinner fibrils with more likelihood of containing collagen type III) collagens [29]. As shown in Fig. 1C\&E, collagen fibrils appear as a range of colours from red/ orange, yellow and green when viewed under polarized light, representing thick, mature and thin, immature collagen, respectively. The background between these coloured fibrils comprised cells, and ECM, which appeared black thus allowing for quantitative morphometric analysis [29]. Fig. 5 shows the morphometric quantitation of mature and immature collagen fibrils. The mature red fibrils synthesis increased with the time in Mesh Tissue Complex (MTC) in both 100 and $200 \mu \mathrm{m}$ increment zone but not in control (Fig. 5 and Supplementary Fig. 1A,B). However, the green, immature collagen fibrils did not change in either MTC or control after 60 and 180 days (Fig. 5 and Suppl Fig. 1C,D). As expected, there was always a greater proportion of mature red fibrils than immature green fibrils in all three groups in both MTC and control areas at both time points (Fig. 5A-H). In all groups, there were no significant differences in the proportion of green immature fibrils in both the MTC and control areas at both times points (Fig. 5E-H). Our results revealed significantly fewer mature red fibrils in the MTC than normal control tissue for all mesh types (overall all 
1
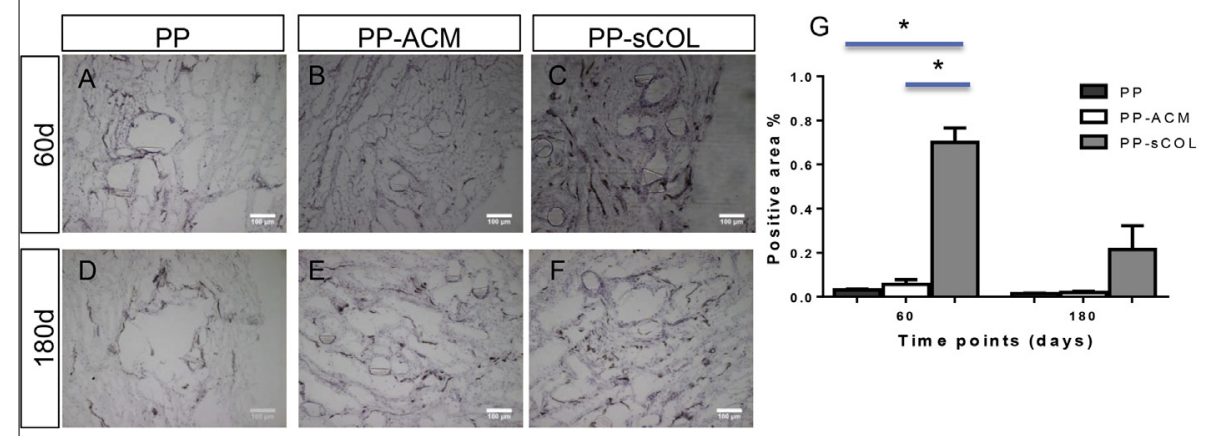

2
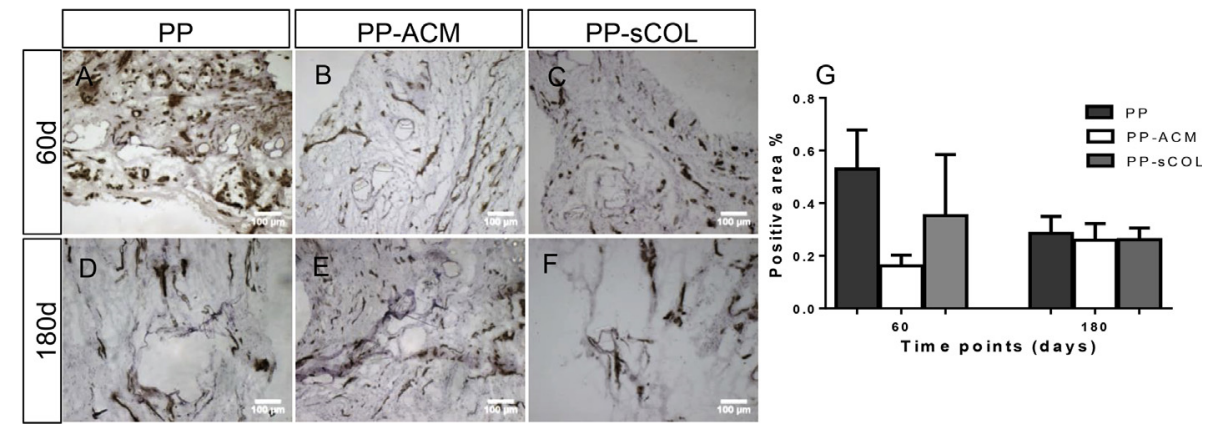

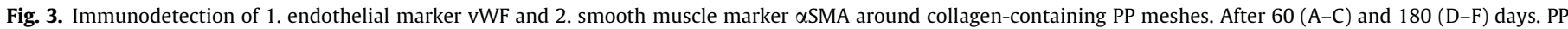

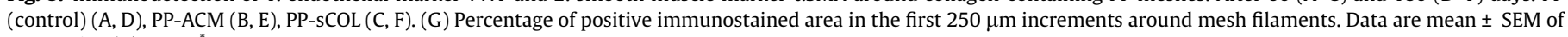
$\mathrm{n}=6$ animals/group. ${ }^{*} \mathrm{P}<0.05$.

$\mathrm{p}<0.001$ ). Mesh-tissue complexes also had significantly less total collagen density (red + green) than normal control tissue regions after 60 days for all three mesh types in both the inner $100 \mu \mathrm{m}$ interface adjacent to the PP filaments and the next $100 \mu \mathrm{m}$ increment zone (Fig. 5I-L; overall all $\mathrm{P}<0.001$ ). There were no significant differences in the proportion of red or green fibrils between any mesh types. However, the total collagen density was significantly greater around the PP-ACM meshes compared with PP $(\mathrm{P}<0.01)$ but not PP-sCOL implant at 60 days; this may in part be due to the presence of remaining slowly degrading collagen tissue layer which was evident in some samples. In general, over the two time points, the proportion of green fibrils remained largely unchanged suggesting a constant rate of renewal of new collagen, but there were significant increases in both the red content $(P<0.01)$ and total collagen density $(P<0.001)$. There were no differences observed in control tissues over time. At 180 days, the total collagen content in both incremental zones of the MTC had increased and was comparable to control tissue $(\mathrm{P}>0.05)$ in both collagen containing PP-ACM and PP-sCOL meshes. In contrast, there was still significantly less mature and immature collagen fibres deposited around the PP mesh at 180 days at both increments $(\mathrm{P}<0.005)$ (Fig. 5J\&L).

\section{Discussion}

The main findings of this study are firstly, that incorporation of collagen into the PP meshes, either as a coating of soluble protein (PP-sCOL) or as a layer of cross-linked collagen tissue (PP-ACM) did not reduce leukocyte accumulation around the mesh filaments compared to unmodified PP meshes at two and six months following vaginal implantation in an ovine model. The majority of inflammatory leukocytes around the filaments were macrophages, and CD163+ M2 macrophages were diminished around PP coated with a sheet of acellular collagen matrix at 60 days but not at 180 days when the entire collagen sheet had been degraded. Secondly, increased microvessel density and therefore vascularization was found around the collagen coated mesh filaments at 60 days, which had resolved by 6 months. Thirdly, there were no differences in the total ECM composition around the implanted meshes using bulk biochemical analyses. However, there were significant differences at the micro-molecular level at the mesh-tissue interface, using birefringence of Sirius Red stained tissues and quantitative morphometric image analysis to track the maturation of newly laid down collagen fibres during the healing phase following mesh implantation. This detailed quantitative analysis demonstrated that mesh implantation reduced the amount of mature collagen around non-collagen containing implanted mesh (PP) for at least 6 months compared to the control regions in the same tissue. More importantly, collagen incorporation in the mesh apparently hastened the maturation of collagen fibres around the mesh filaments, whether it was delivered as a sheet over the mesh or as a soluble protein coating around individual PP filaments. Our analysis revealed that collagen layer-coated mesh delayed the differentiation of infiltrating macrophages to a certain but not all types of M2 wound healing phenotype; it is equally possible that these mesh types could have already gone through the M2 macrophage response and have already begun to influence remodelling to a more mature collagen tissue. Certainly, collagen coated filaments promoted vascularisation between the mesh filaments that accelerated the maturation of newly laid down collagen to produce a connective tissue similar to the original tissue by 6 months.

Macrophages are a heterogeneous population of cells with a range of functional activities depending on their polarization status [30]. In general, they are classified as M1 pro-inflammatory or M2 anti-inflammatory types, both involved in tissue repair and remodelling. Upon activation, they release growth factors which 

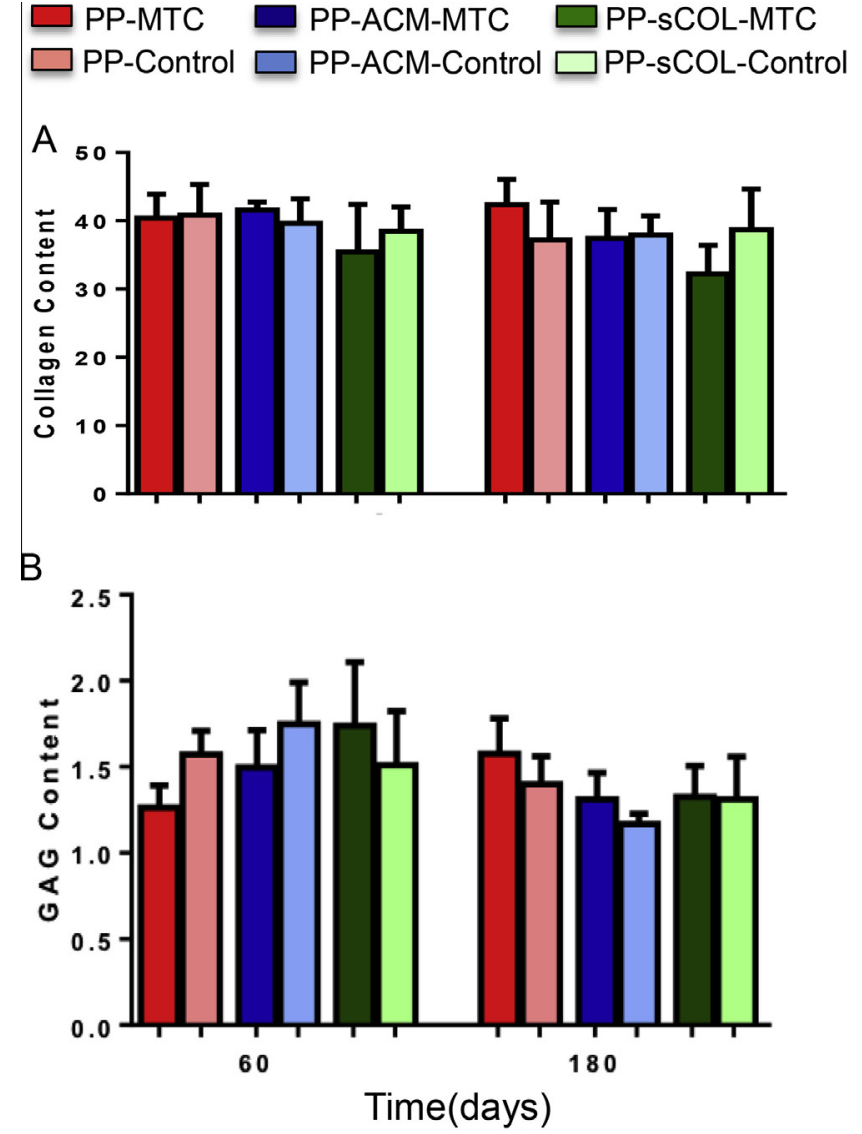

Fig. 4. Extracellular matrix content of collagen - containing mesh implanted tissue. (A) Total collagen and (B) GAG content (\% per dry weight) determined by biochemical assay. Data are mean \pm SEM of $n=6$ animals/group. MTC, Mesh/tissue complex, Control, distal tissue without mesh.

attract fibroblasts and smooth muscle cells to the wound site resulting in collagen production and neo-tissue formation [31]. A vigorous leukocyte response was observed around each of the mesh types, and given the similarity of the percent positive immunostained area, the majority of these leukocytes would appear to be M2 macrophages at the relatively late time points examined in this study. It was expected that the M1 macrophage response would have been seen during the initial wound healing response to these implants. It is also possible that M2 activation could have occurred earlier than 60 days for certain mesh types but was not possible to determine with this type of study design. In addition, several antibodies to M1 macrophages did not cross react with ovine epitopes and could not be assessed. Our finding is consistent with the higher median histology score of macrophage and Foreign Body Giant Cell (FBGC) accumulation observed around the collagen matrix sheet of the PP-ACM mesh in our previous study [17], although low grade infection was also present in this report that might have triggered an M1 type response, deduced from the difference between the CD45 and M2 percent positive areas. Both CD163 and CD206 markers largely showed a similar trend for the three mesh types at both time-points. However, it was difficult to explain the difference in the levels of the two M2 markers at 60 days. CD206 is the C-type mannose receptor 1 and is maintained in resident tissue macrophages independent of cytokine activation [32]. A decrease in CD206 expression is associated with upregulation of pro-inflammatory cytokine production and thus a delay in the ensuing wound healing process. CD163 is a haemoglobin-haptoglobin-scavenger receptor and its expression is not necessarily specific to M2 macrophages. Using double labelling, a sub-population of these CD163 macrophages are associated with the typical classical Thelper1/M1 response [30]. Macrophage polarisation is a dynamic process and it is not entirely clear in the present study what the significance of the transient reduction in CD163+ M2 macrophages means since CD206 M2 macrophages were equally frequent in all implants. Others have demonstrated varying degrees of acute and chronic inflammation following the implantation of collagen or non-collagen coated mesh using different in vivo models $[15,33]$. Similarly, in the ovine vaginal wall no differences in inflammatory response were reported between implantation of coated and non-coated PP mesh [14]. In these studies inflammation was assessed subjectively, based on scoring of macrophages and multinucleated giant cell numbers. In this study we used our new image analysis methods for quantifying positive signals to objectively assess cellular content and vascularization around mesh filaments. Implantation of collagencoated PP mesh in the abdominal wall of rats induced less inflammatory response and granulation tissue at early 7 and 14 day time-points [33] but the M1/M2 status of the macrophages was not reported. For the first time we have used macrophage markers and a quantitative assay evaluating collagen maturation during the tissue response to vaginally implanted PP meshes in an ovine model. We assessed the gradient distribution of macrophages at the filament tissue interface at $100 \mu \mathrm{m}$ increments to more accurately assess the macrophage response as previously reported in a rat model [25].

Since angiogenesis is essential for wound healing we assessed the mesh/tissue complex for neo-vascularization. Our previous evaluation of Polyamide/Gelatin (collagen containing) meshes showed greater neovascularization in a rat abdominal hernia model [25]. In this study, implanted PP-sCOL showed a dramatically increased number of $\mathrm{vWF}^{+}$vessel profiles after 60 days and together with the large accumulation of M2 macrophages suggests enhanced healing takes place around the collagen-coated filament mesh. Similarly a higher vascularization score was observed after 60 and 180 days in PP-sCOL-implanted ovine vagina [17]. In the PP-sCOL group, each mesh filament is coated with soluble noncross-linked highly resorbing collagen, rather than a cross-linked layer of collagen tissue on top of the mesh, allowing the penetration of capillaries and therefore showing greater vascularization. Similar to the dampened M2 macrophage response, the slow degradation of the collagen sheet in PP-ACM may also have prevented vascularization at 60 days. During vascularization, new capillaries develop further to become arterioles and venules through recruitment of smooth muscle cells. In the PP-sCOL MTC, many small fragile capillaries without $\alpha \mathrm{SMA}^{+}(v W F)$ pericytes or mural cells persisted until 60 days but by 180 days these capillaries had acquired mural cells indicating vascular maturation with time. Vessels were assessed at $250 \mu \mathrm{m}$ increments to enable their quantification in neo-tissue as it develops.

Collagen and GAG are two of the main proteins in ECM that are synthesised and remodelled during tissue regeneration after injury and mesh implantation [34]. As the most abundant protein of the ECM, collagen provides strength, integrity and structure to the connective tissue. In the normal process of tissue regeneration and wound healing, fibroplasia and collagen deposition begins in the proliferation zone and continues as the tissue becomes less cellular and remodels to form a mature tissue or scar [35]. During the time of our study the ECM did not change in total collagen and GAG content in the bulk tissue explanted around the three mesh types, which were not different to normal vaginal tissue distant from the implant site. However, by using a quantitative morphometric analyses with Sirius Red birefringence of the newly synthesised collagen tissue, we were able to document the density and maturation of collagen formation at the micro-molecular interface of 


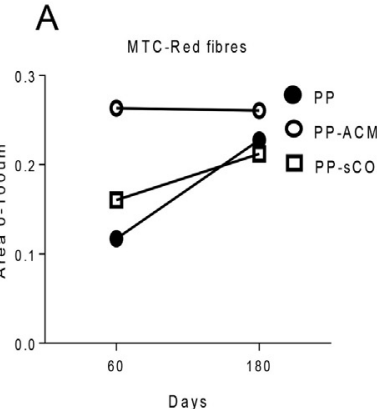

E

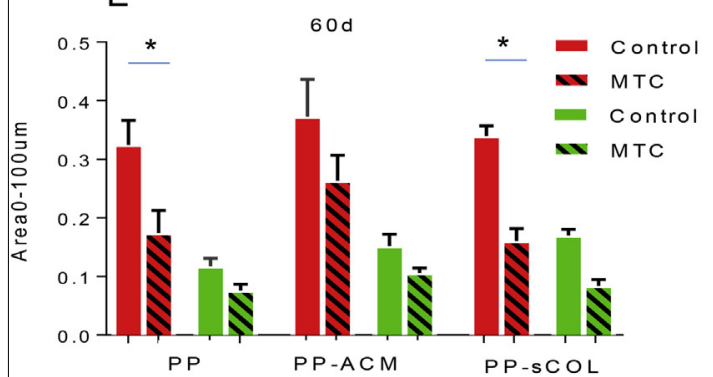

G

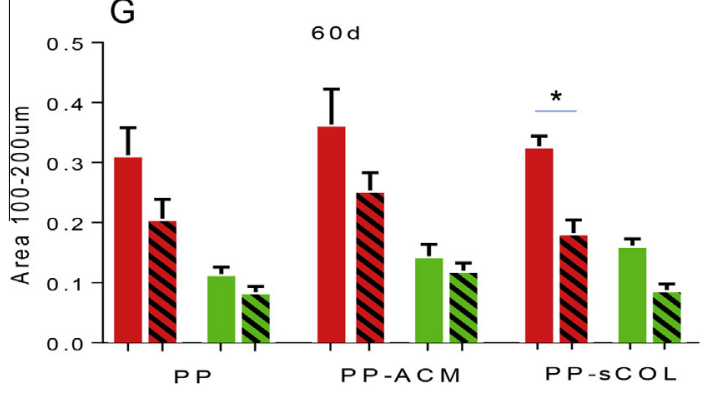

C
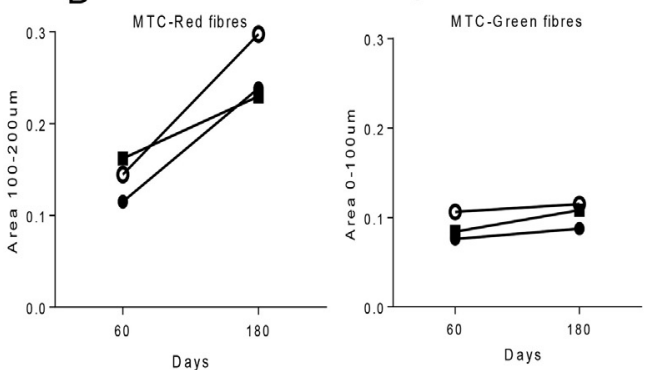

$\mathrm{F}_{*}$

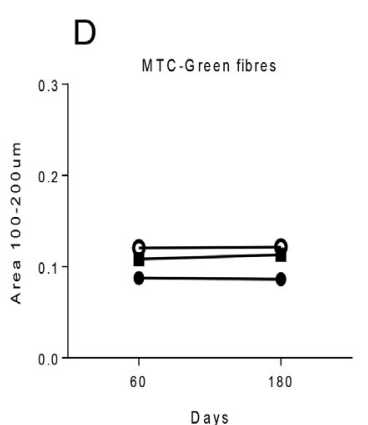

Days
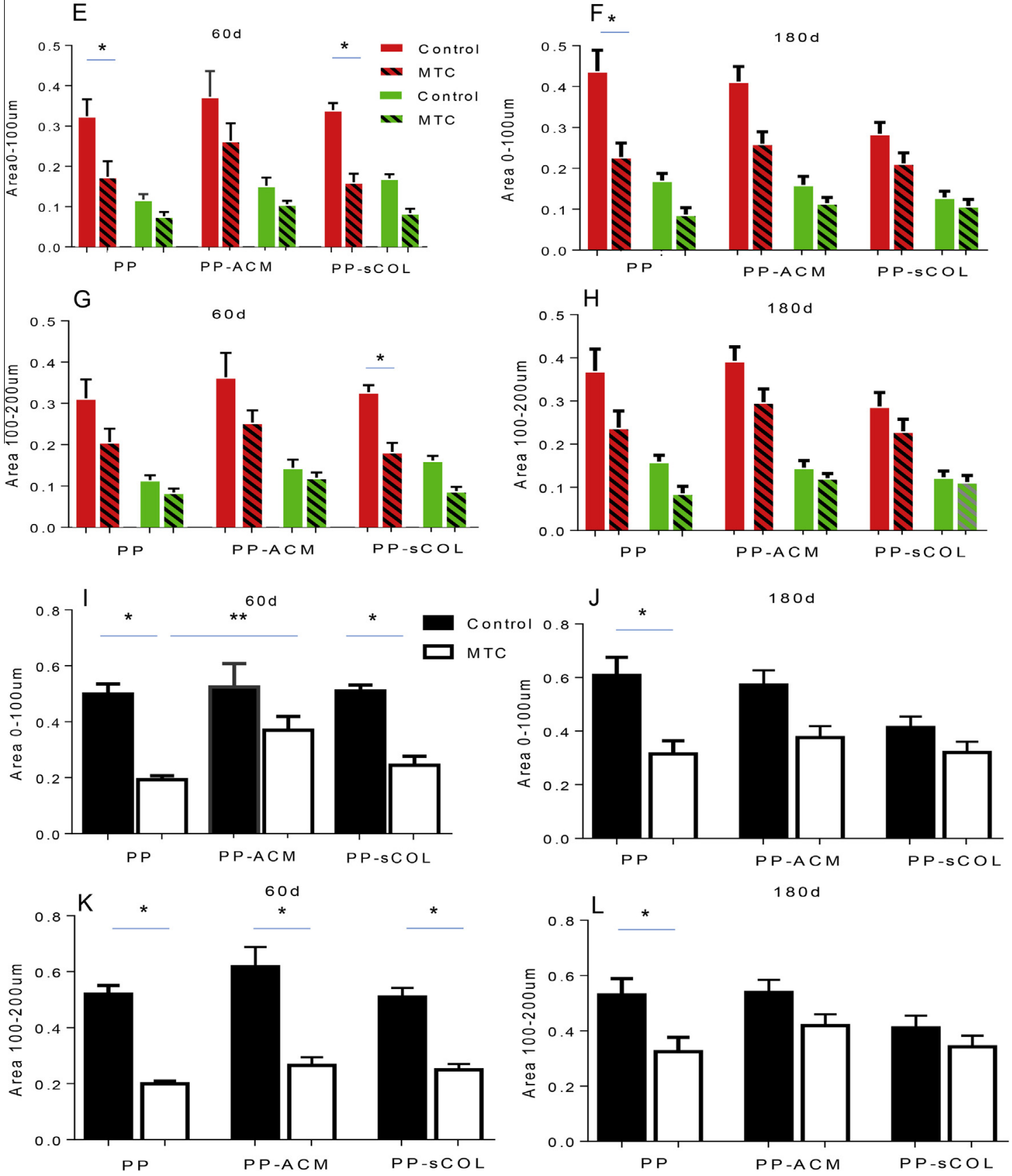

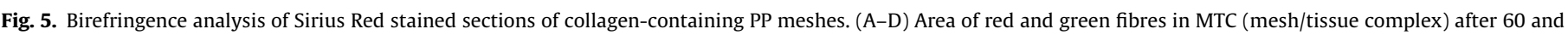

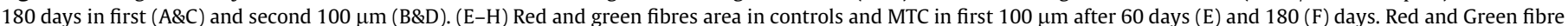

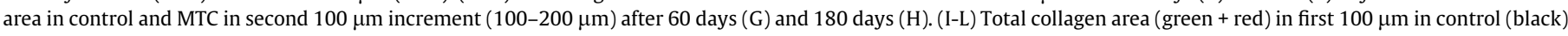

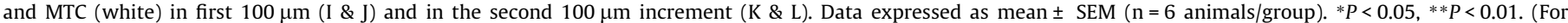
interpretation of the references to colour in this figure legend, the reader is referred to the web version of this article.) 
the mesh implant. We have previously quantified the mature and immature collagen around mesh implants and validated it by Scanning Electron Microscopy (SEM) [36].

The maturity of collagen depends on fibril size. Mature collagen is thick heterodimers while immature ones are thin. Under the polarizing microscope, immature thin collagen fibrils appear green whereas the mature, thick fibrils are red, easily distinguished from black background areas lacking collagen fibre bundles or composed of cells or other ECM proteins. The amount of red mature and green immature collagen fibrils were quantitatively assessed in two larger radii around the mesh filament bundles to better understand the process of new collagen regeneration in relation to the interfacial distance from the mesh filaments. Our data showed consistent and higher levels of mature collagen in control vaginal tissues taken far from the implanted mesh site than around filaments of the three mesh types as expected for uninjured tissue representative of normal tissue with a more mature type of collagen. The Avaulta plus collagen layer is cross-linked with 1-ethyl-3-(3dimethylaminopropyl) carbodiimide (EDAC) which slows collagen degradation (up to 6 month) allowing for longer-term and durable surgical repair. Based on our previous findings there were visible persisting remnants of the cross-linked collagen sheet in 50\% and $17 \%$ of animals in PP-ACM groups after 60 and 180 days, respectively. Comparison of mesh types between the green, red and total birefringent collagen revealed that PP-ACM contained more collagen after 60 days, but this may have been due to the incomplete degradation of the ACM sheet, which could have contributed to the level of mature collagen in the mesh/tissue complex observed at 60 days. While both modified meshes contained collagen, the format of the coating was different. Unlike the PP-sCOL which contained a non cross-linked, non-immunogenic reconstituted soluble atelocollagen, PP-ACM mesh comprised a cross-linked decellularised natural tissue. As such, continuous slowly degrading collagen still present in this group after 60 days would delay the wound tissue response resulting in significantly fewer CD163 positive M2 macrophages (Fig. 3). At 180 days the collagen sheet had completely disappeared [17] and the levels of mature collagen and total collagen around the implants were similar for both types of collagen-containing meshes. Overall, there was a greater proportion of red rather than green birefringent fibrils with time indicating an advanced maturation process in the mesh implanted tissues approaching native vaginal wall tissue. After 180 days both total mature and immature collagen levels in the collagen coated meshes (PP-ACM and PP-sCOL) were closer to native tissue than PP mesh alone, supporting the beneficial effect the incorporation of collagen in the meshes has on the regenerative process.

The meshes used were the most common non-degradable synthetic meshes in use at the time of this study. All three meshes comprised the same polypropylene material, and two contained a collagen coating in two different formats; PP-sCOL in which individual filaments are coated with a non-cross-linked enzymepurified solubilized atelocollagen, providing a fast resorbing layer between individual mesh filaments and the tissue, and PP-ACM, in which a natural decellularised and cross-linked slower degrading collagen tissue is layered on top of the whole mesh implanted. In this study our hypothesis was that PP meshes which are coated with collagen in different ways will induce different responses and that the biocompatibility of the mesh at the mesh tissue interface would be affected by this different format of collagen coating.

Our new more sophisticated quantitative analyses extend our previous study [17] which revealed a trend for increased vascularization in PP-sCOL mesh/tissue complexes using less sensitive histological scoring. With our image analysis method focussed at the interface of the mesh filaments and tissue, we found significantly greater vascularization compared to PP-ACM explants, indicating the increased sensitivity of our quantitative approach. Our birefringent collagen analysis of Sirius red stained tissue explants also demonstrated significant differences between control tissue and mesh explants that were not detected by subjective scoring methods [17], particularly at 60 days where total collagen density was increased for the collagen covered PP mesh, likely due to inclusion of partially degraded collagen at the mesh-tissue interface. Our new quantitative methods applied for the first time to ovine vaginal tissues indicate their sensitivity in detecting differences in tissue response between PP-collagen composite mesh types in assessing chronic inflammation and collagen composition and organisation.

The ideal mesh for POP surgery should not erode into adjacent organs. Mild inflammation promotes neo-vascularization, new collagen deposition and better integration of the mesh. Macrophages play an important role in tissue remodelling and regeneration after mesh implantation and since collagen coating minimizes the contact area between the mesh and tissue, it can directly affect the early macrophage infiltration into the implanted area.

Based on our findings in this study, collagen coating can reduce chronic inflammation and promote the neovascularization and biocompatibility. However the biomechanical properties of the mesh, in particular mesh stiffness should be taken into account as one the main reasons for complications associated with erosion and postsurgery complications. One major limitation of this study was the lack of earlier time points to assess these inflammatory responses and collagen changes and that we were unable to assess the effect of exogenous collagen alone on early tissue responses to implanted meshes. A second limitation was the limited number of antibodies available that cross-react with ovine cells in tissues, particularly to assess the inflammatory M1 macrophage role. A major advantage of this study is using the ovine vagina for implanting mesh due to similarities to human vaginal anatomy. Another strength is the use of non-biased, quantitative measurements of collagen maturity and the leukocyte response to implanted mesh in a field where subjective scoring methods are often used.

\section{Conclusion}

Using the ewe as an experimental model for vaginal prolapse surgery introduces a number of limitations for using advanced molecular techniques. Herein we describe the use of semiquantitative methods for characterizing the host response. We used these for a medium term experiment evaluating the effect of collagen-containing PP meshes for POP surgery. Coating with solubilized atelocollagen showed improved biocompatibility and healing responses, by inducing sustained late neovascularisation and promoting neo-collagen maturation. Coverage of PP with an acellular collagen matrix delayed a partial M2 macrophage response which was likely due to the slow degradation of the exogenous collagen and may have contributed to the high level of mature collagen observed around the mesh 60 days after implantation. We speculate that the M2 macrophage response may result in the secretion of tissue growth factors such as PDGF and TGF $\beta$ to recruit fibroblasts and smooth muscle cells for collagen production and stabilisation of neo-vessels. Further studies are needed to assess the macrophage response and collagen organisation at earlier time-points.

\section{Appendix A. Supplementary data}

Supplementary data associated with this article can be found, in the online version, at http://dx.doi.org/10.1016/j.actbio.2016.05. 010 . 


\section{References}

[1] J.E. Jelovsek, C. Maher, M.D. Barber, Pelvic organ prolapse, Lancet 369 (2007) $1027-1038$.

[2] C. Maher, B. Feiner, K. Baessler, C. Schmid, Surgical management of pelvic organ prolapse in women, Cochrane Database Syst. Rev. 4 (2013). Cd004014.

[3] A.L. Olsen, V. Smith, J. Bergstrom, J. Colling, A.L. Clark, Epidemiology of surgically managed pelvic organ prolapse and urinary incontinence, Obstet. Gynecol. 89 (1997) 501-506.

[4] F.J. Smith, C.D.J. Holman, R.E. Moorin, N. Tsokos, Lifetime risk of undergoing surgery for pelvic organ prolapse, Obstet. Gynecol. 116 (2010) 1096-1100.

[5] D. Altman, T. Vayrynen, M.E. Engh, S. Axelsen, C. Falconer, Anterior colporrhaphy versus transvaginal mesh for pelvic-organ prolapse, N. Engl. J. Med. 364 (2011) 1826-1836.

[6] J. Deprest, F. Zheng, M. Konstantinovic, F. Spelzini, F. Claerhout, A. Steensma, Y. Ozog, D. De Ridder, The biology behind fascial defects and the use of implants in pelvic organ prolapse repair, Int. Urogynecol. J. Pelvic Floor Dysfunct. 17 (Suppl 1) (2006) S16-S25.

[7] J. Deprest, A. Feola, The need for preclinical research on pelvic floor reconstruction, BJOG 120 (2013) 141-143.

[8] R. Liang, S. Abramowitch, K. Knight, S. Palcsey, A. Nolfi, A. Feola, S. Stein, P.A. Moalli, Vaginal degeneration following implantation of synthetic mesh with increased stiffness, BJOG 120 (2013) 233-243.

[9] S. Manodoro, M. Endo, P. Uvin, M. Albersen, J. Vlacil, A. Engels, B. Schmidt, D. De Ridder, A. Feola, J. Deprest, Graft-related complications and biaxial tensiometry following experimental vaginal implantation of flat mesh of variable dimensions, BJOG 120 (2013) 244-250.

[10] FDA Update, Urogynecologic Surgical Mesh: Update on the Safety and Effectiveness of Transvaginal Placement for Pelvic Organ Prolapse (2011).

[11] M. Slack, D. Ostergard, M. Cervigni, J. Deprest, A standardized description of graft-containing meshes and recommended steps before the introduction of medical devices for prolapse surgery. Consensus of the 2nd IUGA Grafts Roundtable: optimizing safety and appropriateness of graft use in transvaginal pelvic reconstructive surgery, Int. Urogynecol. J. 23 (Suppl. 1) (2012) S15-S26.

[12] M.T. Wolf, C.A. Carruthers, C.L. Dearth, P.M. Crapo, A. Huber, O.A. Burnsed, R. Londono, S.A. Johnson, K.A. Daly, E.C. Stahl, J.M. Freund, C.J. Medberry, L.E. Carey, A. Nieponice, N.J. Amoroso, S.F. Badylak, Polypropylene surgical mesh coated with extracellular matrix mitigates the host foreign body response, J. Biomed. Mater. Res. A 102 (2014) 234-246.

[13] J.E. Valentin, A.M. Stewart-Akers, T.W. Gilbert, S.F. Badylak, Macrophage participation in the degradation and remodeling of extracellular matrix scaffolds, Tissue Eng. Part A 15 (2009) 1687-1694.

[14] R. de Tayrac, A. Alves, M. Therin, Collagen-coated vs noncoated low-weight polypropylene meshes in a sheep model for vaginal surgery. A pilot study, Int. Urogynecol. J. 18 (2007) 513-520.

[15] R.K. Huffaker, T.W. Muir, A. Rao, S.S. Baumann, T.J. Kuehl, L.M. Pierce, Histologic response of porcine collagen-coated and uncoated polypropylene grafts in a rabbit vagina model, Am. J. Obstet. Gynecol. 198 (2008) 582.e581582.e587.

[16] J.M. Anderson, A. Rodriguez, D.T. Chang, Foreign body reaction to biomaterials, Semin. Immunol. 20 (2008) 86-100.

[17] A. Feola, M. Endo, I. Urbankova, J. Vlacil, T. Deprest, S. Bettin, B. Klosterhalfen, J. Deprest, Host reaction to vaginally inserted collagen containing polypropylene implants in sheep, Am. J. Obstet. Gynecol. 212 (2015) 474.e471-474.e478.

[18] S.D. Abramowitch, A. Feola, Z. Jallah, P.A. Moalli, Tissue mechanics, animal models, and pelvic organ prolapse: a review, Eur. J. Obstet. Gynecol. Reprod. Biol. 144 (Suppl. 1) (2009) S146-S158.

[19] B.M. Couri, A.T. Lenis, A. Borazjani, M.F. Paraiso, M.S. Damaser, Animal models of female pelvic organ prolapse: lessons learned, Exp. Rev. Obstet. Gynecol. 7 (2012) 249-260.
[20] F.G. Davies, The occurrence of vaginal eversion and allied disorders in fat ewes, Res. Vet. Sci. 11 (1970) 86-90.

[21] S. Ennen, S. Kloss, G. Scheiner-Bobis, K. Failing, A. Wehrend, Histological, hormonal and biomolecular analysis of the pathogenesis of ovine Prolapsus vaginae ante partum, Theriogenology 75 (2011) 212-219.

[22] L.C. Skoczylas, J.P. Shepherd, K.J. Smith, J.L. Lowder, Managing mesh exposure following vaginal prolapse repair: a decision analysis comparing conservative versus surgical treatment, Int. Urogynecol. J. 24 (2013) 119-125.

[23] D. Ulrich, S.L. Edwards, V. Letouzey, K. Su, J.F. White, A. Rosamilia, C.E. Gargett J.A. Werkmeister, Regional variation in tissue composition and biomechanical properties of postmenopausal ovine and human vagina, PLoS ONE 9 (2014) e104972.

[24] M. Endo, I. Urbankova, J. Vlacil, S. Sengupta, T. Deprest, B. Klosterhalfen, A. Feola, J. Deprest, Cross-linked xenogenic collagen implantation in the sheep model for vaginal surgery, Gynecol. Surg. 12 (2015) 113-122.

[25] D. Ulrich, S.L. Edwards, J.F. White, T. Supit, J.A. Ramshaw, C. Lo, A. Rosamilia, J. A. Werkmeister, C.E. Gargett, A preclinical evaluation of alternative synthetic biomaterials for fascial defect repair using a rat abdominal hernia model, PLoS ONE 7 (2012) e50044.

[26] D. Ulrich, K. Su, K.-S. Tan, J.F. White, J.A.M. Ramshaw, C. Lo, A. Rosamilia, J.A. Werkmeister, C.E. Gargett, Human endometrial mesenchymal stem cells modulate the tissue response and mechanical behavior of polyamide mesh implants for pelvic organ prolapse repair, Tissue Eng. Part A 20 (2014) 785798.

[27] J.F. Woessner Jr., The determination of hydroxyproline in tissue and protein samples containing small proportions of this imino acid, Arch. Biochem. Biophys. 93 (1961) 440-447.

[28] I. Barbosa, S. Garcia, V. Barbier-Chassefiere, J.P. Caruelle, I. Martelly, D. PapyGarcia, Improved and simple micro assay for sulfated glycosaminoglycans quantification in biological extracts and its use in skin and muscle tissue studies, Glycobiology 13 (2003) 647-653.

[29] R. Lattouf, R. Younes, D. Lutomski, N. Naaman, G. Godeau, K. Senni, S. Changotade, Picrosirius red staining: a useful tool to appraise collagen networks in normal and pathological tissues, J. Histochem. Cytochem. 62 (2014) 751-758.

[30] M.H.M. Barros, F. Hauck, J.H. Dreyer, B. Kempkes, G. Niedobitek, Macrophage polarisation: an immunohistochemical approach for identifying M1 and M2 macrophages, PLoS ONE 8 (2013) e80908.

[31] R.F. Diegelmann, M.C. Evans, Wound healing: an overview of acute, fibrotic and delayed healing, Front. Biosci. 9 (2004) 283-289.

[32] T. Roszer, Understanding the mysterious M2 macrophage through activation markers and effector mechanisms, Mediators Inflamm. 2015 (2015) 816460.

[33] R.T. Siniscalchi, M. Melo, P.C. Palma, I.M. Dal Fabbro, C. Vidal Bde, C.L. Riccetto Highly purified collagen coating enhances tissue adherence and integration properties of monofilament polypropylene meshes, Int. Urogynecol. J. 24 (2013) 1747-1754.

[34] L. Kati, P.C. Feldner, R. de Castro, E. Kobayashi, M. Sartori, H. Nader, M. Girao, Analysis of glycosaminoglycans in the parametrium and vaginal apex of women with and without uterine prolapse, J. Womens Health 19 (2010) 13411344.

[35] K.S. Midwood, L.V. Williams, J.E. Schwarzbauer, Tissue repair and the dynamics of the extracellular matrix, Int. J. Biochem. Cell Biol. 36 (2004) 1031-1037.

[36] S.L. Edwards, D. Ulrich, J.F. White, K. Su, A. Rosamilia, J.A. Ramshaw, C.E Gargett, J.A. Werkmeister, Temporal changes in the biomechanical properties of endometrial mesenchymal stem cell seeded scaffolds in a rat model, Acta Biomater. 13 (2015) 286-294. 